\title{
Anti-TNF-Alpha-Induced Psoriasis - An Unusual Paradox
}

\author{
Neel Sharma James Lindsay \\ The Royal London Hospital, Barts and London NHS Trust, London, UK
}

\section{Key Words}

Anti-TNF-alpha · Psoriasis · Crohn's disease

\begin{abstract}
Anti-TNF-a agents are currently utilised for the treatment of a vast array of autoimmune conditions including inflammatory bowel disease, rheumatoid arthritis, ankylosing spondylitis and psoriasis. It is however noted that such therapeutic strategies have been linked to the specific induction of cutaneous-based reactions such as dermatitis, erythema multiforme and psoriasis. Here we present the case of a young female patient with Crohn's disease who developed psoriasis following treatment with the anti-TNF-a drug adalimumab and highlight the possible pathogenetic mechanisms involved in such an occurrence.
\end{abstract}

\section{Case Presentation}

A 20-year-old female with extensive terminal ileal and colonic Crohn's disease was initially successfully managed on a drug regimen of prednisolone and mesalazine which helped to induce remission. The patient unfortunately however developed a mesalazine-induced aplastic anaemia. This subsequently resolved with blood product support and withdrawal of this therapy. Her thiopurine methyl transferase levels were normal, so she was commenced on azathioprine. This maintained her disease remission but was associated with neutropenic sepsis, necessitating the withdrawal of the thiopurine therapy with subsequent clinical and endoscopic disease flare. Induction and maintenance infliximab was used to regain remission. This required an infusion dose and frequency escalation to maintain clinical response. After 58 weeks of infliximab therapy, the patient was requiring $10 \mathrm{mg} / \mathrm{kg} 6$ times weekly and hence was transferred to the fully human anti-TNF-a injection adalimumab for loss of response to infliximab. Initially this strategy regained disease control, but due to relapse at $40 \mathrm{mg}$ every other week, the dose was increased step-wise to $80 \mathrm{mg}$ weekly which fortunately maintained remission.

During her treatment with adalimumab the presence of purple-coloured plaque-like lesions primarily on her abdomen were noted (fig. 1 ). 


\begin{tabular}{|c|c|c|c|}
\hline $\begin{array}{r}\text { Case Reports in } \\
\text { Gastuenterology }\end{array}$ & \begin{tabular}{|l} 
Case Rep Gastroenterol 2009;3:404-407 \\
D0I: 10.1159/000257907
\end{tabular} & | Published online: November 21, 2009 & $\begin{array}{l}\text { O } 2009 \text { S. Karger AG, Basel } \\
\text { ISSN 1662-0631 } \\
\text { www.karger.com/crg }\end{array}$ \\
\hline
\end{tabular}

\section{What Is the Diagnosis?}

Figure 1 demonstrates the presence of psoriasis which is presumed to be a result of treatment with adalimumab.

TNF- $\alpha$ has been identified as a key cytokine in the pro-inflammatory pathways that drive diseases such as inflammatory bowel disease and rheumatoid arthritis [1]. AntiTNF- $\alpha$ agents such as infliximab and adalimumab are widely used in the treatment of these conditions and have been shown to have an impact on pro-inflammatory and apoptotic pathways. They are also effective in the treatment of the dermatological and rheumatological manifestations of psoriasis [2]. However, there have been a number of cases reported where such agents may be responsible for new onset or worsening of psoriasis. A study of 126 patients who underwent treatment with anti-TNF- $\alpha$ inhibitors demonstrated evidence of psoriasis in 76 individuals, palmoplantar pustular psoriasis in 37 cases and psoriasis of the nails in 6 cases [3]. There is mounting evidence that a key innate immune pathway for triggering common human autoimmune diseases, including psoriasis, involves plasmacytoid dendritic cell precursors and type 1 interferon (IFN) production. A recent study involving immunohistochemical staining of skin biopsy specimens for myxovirus-resistance protein $\mathrm{A}(\mathrm{MxA}$, a surrogate marker for lesional type 1 IFN activity) demonstrated increased staining in TNF- $\alpha$ inhibitor-induced psoriasis compared with psoriasis vulgaris [4]. Research suggests that a psoriasiform eruption during anti-TNF- $\alpha$ treatment seems to be a class effect without any confirmed predisposing factors and does not specifically require treatment discontinuation [5]. Further studies are however needed to determine the exact mechanism of and appropriate treatment for anti-TNF- $\alpha$-induced psoriasis.

\section{Acknowledgements}

Dr. James Lindsay has received unrestricted educational grants and received honoraria for consultancy services from Schering-Plough and Abbott UK. He is on the advisory board of ScheringPlough, Abbott UK, and Shire UK. 
Fig. 1. Presence of psoriasis which is presumed to be a result of treatment with adalimumab.

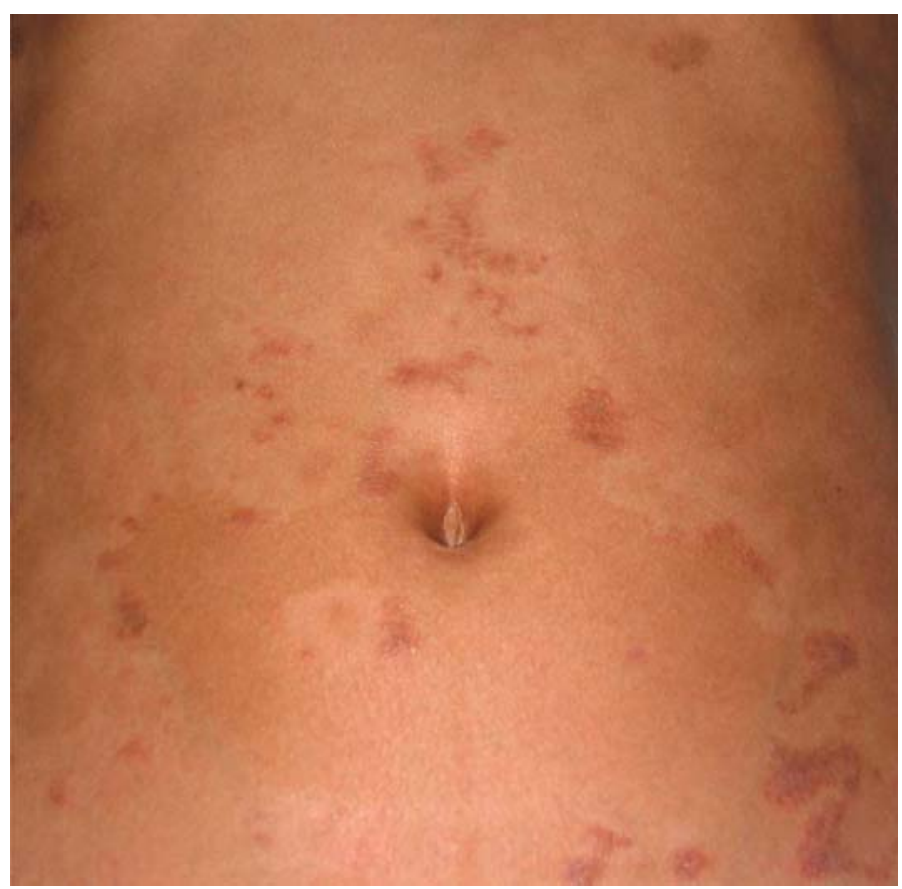




\section{References}

1 Victor FC, Gottlieb AB: TNF-alpha and apoptosis: implications for the pathogenesis and treatment of psoriasis. J Drugs Dermatol 2002;1:264-275.

2 Aslanidis S, Pyrpasopoulou A, Douma S, Triantafyllou A: Tumor necrosis factor$\alpha$ antagonist-induced psoriasis: yet another paradox in medicine. Clin Rheumatol 2008;27:377-380.

-3 Wollina U, Hansel G, Koch A, et al: Tumor necrosis factor-alpha inhibitorinduced psoriasis or psoriasiform exanthemata: first 120 cases from the literature including a series of six new patients. Am J Clin Dermatol 2008;9:347.

4 de Gannes GC, Ghoreishi M, Pope J, Russell A, Bell D, Adams S, Shojania K, Martinka M, Dutz JP: Psoriasis and pustular dermatitis triggered by TNF-a inhibitors in patients with rheumatologic conditions. Arch Dermatol 2007;143:223-231.

5 Pirard D, Arco D, Debrouckere V, Heenem M: Anti-tumor necrosis factor alphainduced psoriasiform eruptions: three further cases and current overview. Dermatology 2006;213:182-186. 